\title{
SUGGESTIONS FOR INFRARED OBSERVATIONS OF THE SOLAR CORONA
}

\section{P. Swings}

The war has caused a number of astronomers to study techniques and instruments which at first thought seem far from their usual sphere of interest. Many of those temporarily exiled from astronomy see how their newly acquired skills can be used to good advantage when normal scientific life is resumed after the war. For example, postwar astronomy will most likely be greatly influenced by the electronic and radio techniques applied to war problems. This will also be true, although probably to a less extent, in many other fields, such as optics, infrared spectroscopy, and mechanics. It may well be that the value of these fresh techniques to postwar astronomy will more than balance the decrease in scientific production during the war.

For example, we may expect postwar observers of the solar corona, with the coronagraph or during eclipses, to devote more attention to the infrared region of the spectrum beyond $1.35 \mu$, the present limit of photographic emulsions. Observation of infrared coronal lines would help in elucidating the whole physics of coronal emission. The observation by B. Lyot in 1936 of the infrared doublet $\lambda 10746.80-\lambda 10797.95$ was of great importance in bringing about the remarkable interpretation of the coronal lines by B. Edlén : ${ }^{1}$ these two infrared lines are actually due to atoms of iron twelve times ionized. New coronal lines and their identifications would add valuable information concerning the chemical constitution, ionization limits, and eventually the excitation mechanisms of the corona. In the isoelectric sequences $3 \mathrm{~s}^{2} 3 \mathrm{p}, 3 \mathrm{~s}^{2} 3 \mathrm{p}^{5}, 2 \mathrm{~s}^{2} 2 \mathrm{p}$, and $2 \mathrm{~s}^{2} 2 \mathrm{p}^{5}$ there are several predicted infrared transitions ${ }^{2} \mathrm{P}-{ }^{2} \mathrm{P}$. Similarly the isoelectric sequences $3 \mathrm{~s}^{2} 3 \mathrm{p}^{2}, 3 \mathrm{~s}^{2} 3 \mathrm{p}^{4}, 2 \mathrm{~s}^{2} 2 \mathrm{p}^{2}$, and $2 \mathrm{~s}^{2} 2 \mathrm{p}^{4}$ should also reveal additional forbidden infrared transitions of the ${ }^{1} \mathrm{D}-{ }^{3} \mathrm{P}$ and ${ }^{3} \mathrm{P}-{ }^{3} \mathrm{P}$ types.

${ }^{1} Z s . f . A p ., 22,30,1942$; for a review of Edlén's work, see $A p . J ., 98$, $116,1943$. 
Infrared observations are of course handicapped by the lack of suitable photographic emulsions and by the strong atmospheric absorption bands. Whether or not the evaporographic method developed by Czerny and his collaborators ${ }^{2}$ can be rendered sufficiently sensitive to show coronal lines is a matter that astronomers should settle! After all the evaporographic technique,. like photography, is cumulative, and astronomers should be able to make excellent use of it, despite its technical difficulties. So far as we know, even the solar spectrum has not yet been "evaporographed" in the region 1.35 to $24 \mu$, although this project does not seem to be too complicated. Adel's important investigations of the infrared spectrum with the thermopile ${ }^{3}$ give a good general idea of what to expect on a solar "evaporogram."

If bolometric or thermoelectric receivers are used, the tremendous recent progress in amplifiers, oscillographs, etc., will be of considerable help. Although coronal variations must be taken into account, observations with a coronagraph may be performed fairly slowly (i.e., a short spectral region at a time), and the usual types of thermopiles and sensitive galvanometers with periods from one to five seconds may be used with the maximum amplifications compatible with the Brownian motion.

Much work will still have to be reserved for total eclipses, during which the factor of speed is essential. This at once excludes the usual multijunction thermopiles and most sensitive galvanometers. The receiver may be a single strip bolometer or, possibly, a single junction thermocouple. A quick response (period of the order of $0.2 \mathrm{sec}$.) is obtainable, and special galvanometers, such as the micro Moll, may then be used. The spectrum may be made to appear on a long persistence oscilloscope screen. A persistence of one minute is possible, so that the interesting portions of the infrared spectrum could be located, adjusted for the optimum resolution, and either photographed or recorded on some adaptation of a sound power level recorder. Industrial infrared spectrographs have been designed

\footnotetext{
2 Zs. f. Phys., 53, 1, $1929 ;$ 108, 85, 1937.

3 Ap. J., 93, 506, 509, 1941 ; 94, 375, 379, 449, 451, 1941 ; 96, 239, 1942 ; 97, 190, 1943.
} 
embodying these principles and there would be no new technical difficulty in constructing a similar astronomical instrument.

Whether any coronal emission line shining between telluric absorption bands would have enough intensity to be detected, only the actual observation can tell ; guesses could be highly dan- gerous to progress!

Such an infrared spectrograph could be readily adapted to stellar spectroscopy. The far infrared region doubtless holds treasures for the observer despite the atmospheric absorption bands.

Pasadena, California

March 9, 1944 